\title{
Comfort and exertion while using filtering facepiece respirators with exhalation valve and an active venting system among male military personnel
}

\author{
Melvin Seng ${ }^{1,2, *}$, MBBS, MPH, Liang En $\underline{\text { Wee }}^{2,3, *}$, MBBS, Xiahong Zhao ${ }^{4}$, BSc(Hons), Alex $R \underline{\text { Cook }}^{4,5}$, PhD,
} Sin Eng $\underline{\text { Chia }}^{4}$, MBBS, MD, Vernon J $\underline{\operatorname{Le}}^{2,4}$, MBBS, PhD

\begin{abstract}
INTRODUCTION This study aimed to determine if disposable filtering facepiece respirators (FFRs) that come with an exhalation valve (EV) and a novel active venting system (AVS) provided greater perceived comfort and exertion when compared to standard N95 FFRs without these features among male military personnel performing prolonged essential outdoor duties.

METHODS We used a randomised open-label controlled crossover study design to compare three FFR options: (a) standard FFR; (b) FFR with EV; and (c) FFR with EV+AVS. Male military personnel aged between 18 and 20 years completed a questionnaire at the start of outdoor duty (baseline), after two hours of standardised non-strenuous outdoor duty and after 12 hours of duty divided into two-hour work-rest cycles. Participants rated the degree of discomfort, exertion and symptoms using a five-point Likert scale. The association between outcomes and types of FFR was assessed using a multivariate ordered probit mixed-effects model.

RESULTS For a majority of the symptoms, study participants gave FFR with EV and FFR EV+AVS significantly better scores than standard FFR. Both FFR with EV and FFR with EV+AVS had significantly less discomfort (FFR with EV+AVS: 91.1\%; FFR with EV: 57.6\%) and exertion (FFR with EV+AVS: 83.5\%; FFR with EV: 34.4\%) than standard FFR. FFR with EV+AVS also had significantly better scores for exertion (53.4\%) and comfort (39.4\%) when compared to FFR with EV. CONCLUSION Usage of FFR with EV+AVS resulted in significantly reduced symptoms, discomfort and exertion when compared to FFR with EV and standard FFR.
\end{abstract}

Keywords: active venting system, comfort, exertion, exhalation valves, N95 respirator

\section{INTRODUCTION}

Air pollution, specifically particulate matter (PM), is established as a major cause of cardiorespiratory morbidity and mortality. ${ }^{(1)}$ In Southeast Asia, transboundary smoke haze episodes have been a recurrent cause of air pollution. Due to poorly controlled biomass and peat burning in parts of Indonesia, the increase in airborne PM can be a significant cause of air pollution, especially during the months of August to December. ${ }^{(2)}$ During these haze periods, $\mathrm{PM}_{2.5}$ and $\mathrm{PM}_{10}$ concentrations can be significantly elevated..$^{(3)}$ This can translate to an increase in sickness absenteeism for haze-related conditions. ${ }^{(4,5)}$ During haze periods, the local health authorities in Singapore issue health advisories recommending the postponement or reduction of non-essential outdoor activities, as well as the use of filtering facepiece respirators (FFRs) for personnel doing essential outdoor duties, depending on the level of air pollution. ${ }^{(6)}$

Respiratory protection can be achieved via engineering and administrative controls, as well as through the use of personal protective equipment (PPE), such as disposable FFRs, to reduce personal exposure; this is especially important for outdoor workers. Among FFRs, the N95 FFRs are most commonly recommended and widely used. ${ }^{(7)}$ However, the protective value of PPE has to be weighed against the physiological and psychological burden it imposes on the user. Respirators have been shown to provide beneficial cardiovascular effects through reducing exposure to particulate air pollution, ${ }^{(8)}$ but they can also have physiological and psychological effects. After one hour of FFR use, carbon dioxide $\left(\mathrm{CO}_{2}\right)$ levels, temperature and humidity can be significantly elevated in the dead space within the FFR even as oxygen levels are lowered to levels below ambient workplace standards. ${ }^{(9,10)}$ Discomfort and exertion have been shown to increase over time with continual respirator use. ${ }^{(11)}$ To address this issue, an exhalation valve (EV) has been introduced into various FFR models to help dissipate the heat, humidity and $\mathrm{CO}_{2}$ that accumulate within the dead space. Review of the existing literature has shown that EVs may decrease exhalation resistance and improve dead space heat dissipation, but there is little effect on dead space humidity or other physiological variables. ${ }^{(12)}$ Recently, a novel active venting system (AVS) that includes a lightweight battery-operated fan has been developed to further improve ventilation of the dead space. However, research on the impact of AVS is limited at present, and to the best of our knowledge, there is currently no published study on FFRs with EV+AVS. As comfort and exertion can impact compliance with

\footnotetext{
${ }^{1}$ Preventive Medicine, National University Health System, ${ }^{2}$ Biodefence Centre, Force Medical Protection Command, Singapore Armed Forces Medical Corps, ${ }^{3}$ SingHealth Internal Medicine, ${ }^{4}$ Saw Swee Hock School of Public Health, National University of Singapore, National University Health System, ${ }^{5}$ Yale-NUS College, National University of Singapore, Singapore *These two authors contributed equally to the study.

Correspondence: A/Prof Vernon Jian Ming Lee, Head, Biodefence Centre, Force Medical Protection Command, Singapore Armed Forces Medical Corps, Singapore 778910. vernonljm@hotmail.com
} 

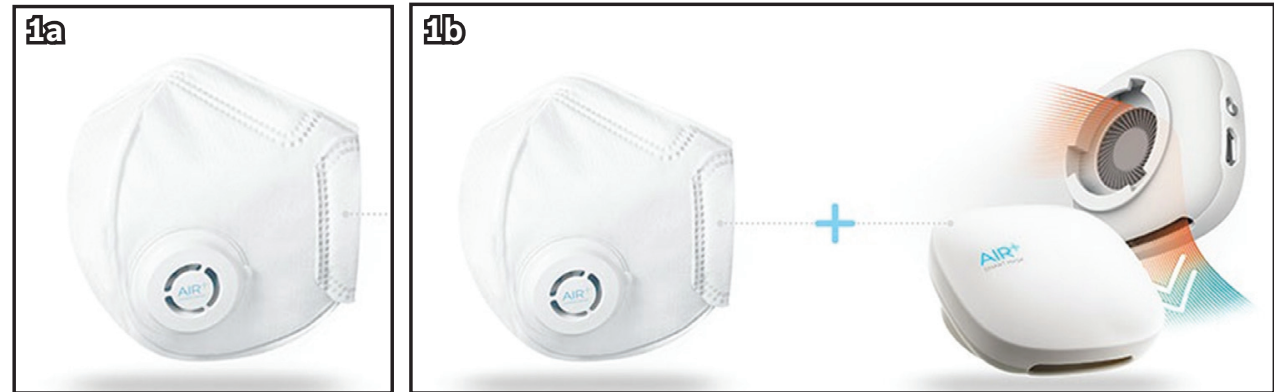

Fig. 1 Photographs show (a) the filtering facepiece respirator with exhalation valve and (b) the active venting system add-on.
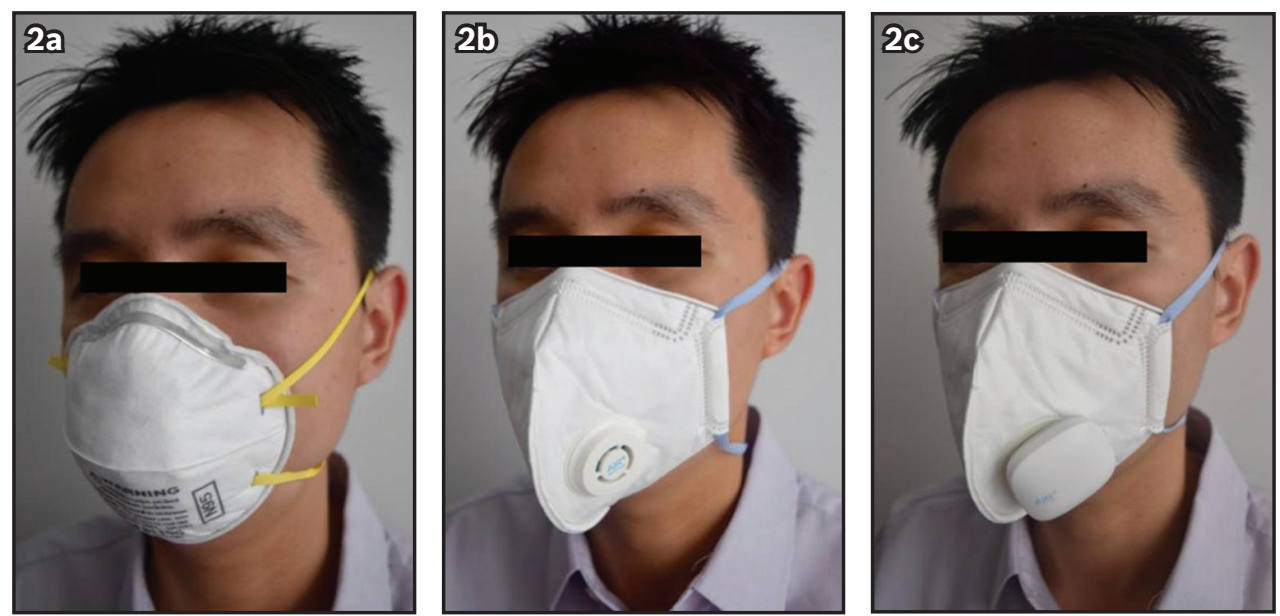

Fig. 2 Photographs show personnel wearing (a) standard filtering facepiece respirator (FFR); (b) FFR with exhalation valve (EV); and (c) FFR with EV+active venting system. The FFRs were the National Institute for Occupational Safety and Health-approved N95 particulate FFRs. Respirators were self-checked to determine good fit prior to usage according to the manufacturer's instructions.

FFR usage, further research into the usage of FFR with AVS+EV is warranted.

In this study, we assessed self-reported discomfort, exertion and symptoms from wearing FFR with and without EV, and FFR with EV+AVS using a crossover trial methodology among military personnel who were required to wear suitable PPEs while performing essential outdoor duties. We also aimed to determine whether FFR with EV and/or FFR with EV+AVS provided better perceived comfort and user tolerance on extended periods of use, as might be necessitated during long-lasting air pollution events.

\section{METHODS}

This study was performed among 120 healthy military personnel of the Singapore Armed Forces (SAF) in November 2015. Informed verbal consent was taken from all participants and study participation was voluntary. The study was approved as part of an operational test and evaluation project by the Joint Medical Committee 01/16 under institutional research guidelines.

The inclusion criteria were personnel serving on active duty with a unit and assigned to perform essential guard duties. Personnel were excluded if: (a) they had cough, fever, respiratory symptoms or any intercurrent illnesses; (b) they were receiving any regular medications at the time of the study; or (c) they had asthma, heart diseases or other lung diseases.

Three types of FFRs were used in the study: (a) standard N95 FFR without EV and AVS (henceforth referred to as standard FFR; 3M 8210 model); (b) N95 FFR with EV (henceforth referred to as
FFR with EV; AIR+ Smart N95 mask [Fig. 1a]; Innosparks Pte Ltd, Singapore); and (c) N95 FFR with EV and AVS (henceforth referred to as FFR with EV+AVS; AIR+ Smart mask with a novel AVS - the Air+ microventilator [Fig. 1b]; Innosparks Pte Ltd, Singapore).

The AVS comprised a one-way valve (i.e. EV), a blower and a battery in a lightweight housing unit (AVS weight $19 \mathrm{~g}$ ) that could be attached to the FFR with negligible deformation of the filter. The blower vented air from the FFR and was powered by a rechargeable battery. In computational fluid dynamics models, the AVS has been shown to potentially reduce the $\mathrm{CO}_{2}$ levels within the dead space of the FFR to near-ambient levels. ${ }^{(13)}$

We used a randomised open label controlled crossover study design to compare perceived user comfort and tolerance of the three FFR options: (a) standard FFR; (b) FFR with EV; and (c) FFR with EV+AVS (Fig. 2). Participants took part in the study on three days, with a washout period of 24 hours between each study day. They were randomised to wear the standard FFR, FFR with EV or FFR with EV+AVS on either of the three days, such that the day on which they wore each option was assigned based on a random number generator. Participants were asked to complete a symptom questionnaire at the beginning of each study day, after two hours of standardised non-strenuous outdoor activity on the study day and at the end of the study day.

Participants were involved in guard duty at various military camps/installations for a total of 12 hours that was divided into two-hour work-rest cycles (i.e. two hours of guard duty followed by two hours of rest indoors, followed by successive work-rest 
cycles). During guard duty, the participants performed prowling duty at a regular slow walk around a standardised circuit with weapons and wearing the FFR; during rest periods, they rested in air-conditioned accommodation without wearing the FFR. Batteries of the AVS were recharged during the participants' rest periods. The protocol selected was based on the usual protocols in the SAF for personnel performing guard duty, which is considered a non-strenuous, essential outdoor duty.

The study questionnaire was completed at three time points - at the start of guard duty (baseline), after the first two-hour duty stint and at the end of the 12-hour duty stint. Participants were asked to rate, on a Likert scale of 1-5 (1: completely disagree; 5: completely agree), whether they experienced the following symptoms while wearing the various FFRs: headache; giddiness; tiredness; difficulty breathing; difficulty walking; sensation of warmth within the mask; sweating within the mask; itchiness within the mask; and uncomfortable tightness around the mask seal. Participants were also asked to indicate the degree of discomfort and exertion experienced when wearing the mask using the five-point Likert scale. The questionnaire was modified from similar questionnaires that were used to evaluate userperceived discomfort during FFR use, ${ }^{(9)}$ with minor modifications made after a pilot study was conducted to evaluate user feedback and perceptions of the FFR options $(n=5)$. Participants were instructed to don the FFR at all times when performing duties outdoors and to remove them when resting indoors.

The calculation of sample size depended on a presumed probability distribution of the comfort level of various FFR options. In view of our assumptions on the probability distribution of the comfort level, and power $=0.80$ and $\alpha=0.05$, it was ascertained that a sample size of at least 115 participants would be needed in each group to detect a significant difference. The final sample size was rounded up to 120 participants for this study.

To describe the trend of perceived user discomfort and tolerance of the three FFR options, the distribution of Likert scores was calculated at various time points. We developed a multivariate ordered probit mixed-effects model to determine the impact of various FFR options on symptom severity, and exertion and comfort levels. The model was a type of mixed-effect generalised linear model that was designed for ordinal repeated measurements. ${ }^{(14,15)}$ The outcome variable was the repeated measurements of perceived symptoms, exertion and comfort scores, and the predictors were FFR options, sampling periods (at baseline, after two hours and after 12 hours), interaction between sampling periods and FFR options, and smoking status. Nonsignificant demographic variables, such as age and ethnicity, were excluded from the multivariate model at a significance level of $p<0.05$. Specifically, the distribution of response scores in the model was:

$$
\begin{gathered}
y_{i j}=\mathrm{k} \text { if } \mathrm{z}_{i j} \in\left(\theta_{k-1} \theta_{k}\right), \mathrm{k}=1,2,3,4 \text { and } 5 \\
\mathrm{z}_{i j} \sim \mathrm{N}\left(\mu_{i j}, 1\right) \\
\mu_{i j}=\boldsymbol{\beta}^{\top} \mathbf{X}_{i j}+\varepsilon_{i}
\end{gathered}
$$

where $y_{i j}$ is the $j$ th observed response score for individual $i ; z_{i j}$ is the latent variable that represents the observed response score on the normal distribution bounded by the ordered thresholds $\theta_{k}$
Table I. Demographics of study participants $(n=114)$.

\begin{tabular}{|ll|}
\hline Variable & No. (\%) \\
\hline Smoking status & $75(65.8)$ \\
\hline Non-smoker & $2(1.8)$ \\
\hline Ex-smoker (stopped $\geq 3 \mathrm{mth})$ & $37(32.5)$ \\
\hline Current smoker & \\
\hline Ethnicity & $75(65.8)$ \\
\hline Chinese & $26(22.8)$ \\
\hline Malay & $6(5.3)$ \\
\hline Indian & $7(6.1)$ \\
\hline Other & $105(92.1)$ \\
\hline Vocation & $9(7.9)$ \\
\hline Infantry trooper & \\
\hline Infantry sergeant (commander) & \\
\hline
\end{tabular}

determining observations; $\mu_{i j}$ is the mean of the latent variable; $\mathbf{X}_{i j}$ is the covariates matrix (consisting of types of masks, time points, interaction between time points, and types of masks, and smoking); and $\varepsilon_{i}$ is the random effect term for each individual.

Model parameters were estimated using the Markov chain Monte Carlo method, with 50,000 iterations and a thin of 5 after a burn-in period of 1,000 iterations. Trace plots and Geweke's convergence diagnostic were used to assess the convergence of the model. ${ }^{(16)}$ Statistical analysis was performed using the rjags extension in $\mathrm{R}$ version 3.2.1 (R Foundation for Statistical Computing, Vienna, Austria). ${ }^{(17,18)}$

\section{RESULTS}

In total, 114 of 120 military personnel recruited completed the study (participation rate of $95.0 \%$ ). Six individuals were excluded from the final analysis because of incomplete data. Demographic characteristics of the study participants are shown in Table I. All participants were male, aged 18-20 years and had no medical comorbidities. About one-third (32.5\%) of our participants were current smokers and two-thirds (65.8\%) were of Chinese ethnicity. User compliance with the mask was extremely good, with a majority ( $\mathrm{n}=112 ; 98.2 \%$ ) of individuals wearing the mask for the full stipulated duration of the study.

The estimated percentages of participants having lower scores (i.e. less symptoms, discomfort or exertion) versus the reference group are presented in Table II and Fig. 3. The posterior means of regression coefficients with 95\% credible intervals (Crls) are shown in Table III. Ever-smokers tended to have more breathing problems (posterior mean 1.92; 95\% Crl 0.11-3.81). 83.5\% of participants using FFR with EV+AVS and $34.4 \%$ of participants using FFR with EV had significantly less exertion when compared to participants using FFR alone. Among participants using FFR with EV+AVS, 53.4\% had significantly less exertion when compared to participants using FFR with EV. In terms of exertion after 12 hours of mask usage, the posterior mean of FFR with EV+AVS was -1.18 (95\% Crl -1.47 to -0.89$)$ when compared to -0.45 (95\% $\mathrm{Crl}-0.74$ to -0.16$)$ for those using FFR with EV. Similarly, 91.1\% of participants using FFR with EV+AVS and $57.6 \%$ of participants using FFR with EV had significantly less discomfort 
Table II. Estimated percentages of participants having less perceived symptoms, discomfort and exertion associated with the usage of various FFR options, with and without EV or AVS, at 12 hours $(n=114)$.

\begin{tabular}{|c|c|c|}
\hline \multirow[t]{2}{*}{ Symptom* } & \multicolumn{2}{|l|}{$\%(95 \% \mathrm{Crl})^{+}$} \\
\hline & FFR with EV and FFR with EV+AVS vs. standard FFR & FFR with EV+AVS vs. FFR with EV \\
\hline \multicolumn{3}{|l|}{ Headache } \\
\hline FFR alone ${ }^{\ddagger}$ & - & - \\
\hline FFR with EV & $23.2(0.0-47.4)$ & - \\
\hline FFR with EV+AVS & $56.7(33.3-77.2)$ & $33.9(9.6-57.9)$ \\
\hline \multicolumn{3}{|l|}{ Giddiness } \\
\hline FFR alone $e^{\ddagger}$ & - & - \\
\hline FFR with EV & $20.3(0.0-43.0)$ & - \\
\hline FFR with EV+AVS & $52.7(30.7-71.9)$ & $32.9(10.5-55.3)$ \\
\hline \multicolumn{3}{|l|}{ Tiredness } \\
\hline FFR alone ${ }^{*}$ & - & - \\
\hline FFR with EV & $45.7(22.8-67.5)$ & - \\
\hline FFR with EV+AVS & $67.0(44.7-81.6)$ & $22.3(0.0-44.7)$ \\
\hline \multicolumn{3}{|l|}{ Difficulty breathing } \\
\hline FFR alone ${ }^{\ddagger}$ & - & - \\
\hline FFR with EV & $42.6(17.5-67.5)$ & - \\
\hline FFR with EV+AVS & $89.2(74.6-94.6)$ & $56.5(31.6-81.6)$ \\
\hline \multicolumn{3}{|l|}{ Difficulty walking } \\
\hline FFR alone $e^{\ddagger}$ & - & - \\
\hline FFR with EV & $23.0(1.8-44.7)$ & - \\
\hline FFR with EV+AVS & $38.4(17.5-57.9)$ & $16.1(0.0-37.7)$ \\
\hline \multicolumn{3}{|c|}{ Sensation of warmth within the mask } \\
\hline FFR alone ${ }^{\ddagger}$ & - & - \\
\hline FFR with EV & $46.4(18.4-73.7)$ & - \\
\hline FFR with EV+AVS & $91.9(88.6-95.6)$ & $80.8(55.3-93.0)$ \\
\hline \multicolumn{3}{|c|}{ Sweating within the mask } \\
\hline FFR alone ${ }^{*}$ & - & - \\
\hline FFR with EV & $52.6(26.3-78.1)$ & - \\
\hline FFR with EV+AVS & $89.7(84.2-94.7)$ & $60.8(33.3-86.8)$ \\
\hline \multicolumn{3}{|c|}{ Itchiness within the mask } \\
\hline FFR alone ${ }^{*}$ & - & - \\
\hline FFR with EV & $53.5(28.9-77.2)$ & - \\
\hline FFR with EV+AVS & $82.1(63.2-91.2)$ & $33.3(8.8-58.8)$ \\
\hline \multicolumn{3}{|c|}{ Uncomfortable tightness of mask seal } \\
\hline FFR alone ${ }^{\ddagger}$ & - & - \\
\hline FFR with EV & $74.9(50.0-91.2)$ & - \\
\hline FFR with EV+AVS & $92.4(87.7-95.6)$ & $40.4(14.0-66.7)$ \\
\hline \multicolumn{3}{|l|}{ Discomfort } \\
\hline FFR alone ${ }^{\ddagger}$ & - & - \\
\hline FFR with EV & $57.6(34.2-80.7)$ & - \\
\hline FFR with EV+AVS & $91.1(73.7-98.2)$ & $39.4(14.9-64.0)$ \\
\hline \multicolumn{3}{|l|}{ Exertion } \\
\hline FFR alone $e^{*}$ & - & - \\
\hline FFR with EV & $34.4(11.4-57.9)$ & - \\
\hline FFR with EV+AVS & $83.5(65.8-97.4)$ & $53.4(30.7-75.4)$ \\
\hline
\end{tabular}

*Symptoms, comfort and exertion were rated on a 5-point Likert scale (1: less symptoms/discomfort/exertion; 5: most symptoms/discomfort/exertion). +Estimated percentages of participants having lower scores (i.e. less symptoms, discomfort or exertion).

when compared to those using FFR alone. $39.4 \%$ of participants using FFR with EV+AVS had significantly less discomfort when compared to participants using FFR with EV. For discomfort after 12 hours of mask usage, the posterior mean of FFR with
EV+AVS was $-1.26(95 \% \mathrm{Crl}-1.55$ to -0.96$)$ when compared to $-0.77(95 \% \mathrm{Crl}-1.06$ to -0.48$)$ for those using FFR with EV.

For the majority of symptoms, study participants gave FFR with EV and FFR with EV+AVS significantly better scores 
Table III. Posterior means with $95 \%$ Crl of the regression coefficients for perceived symptoms, discomfort and exertion associated with the usage of various FFR options, with and without EV or AVS, at two hours and 12 hours $(n=114)$.

\begin{tabular}{|c|c|c|c|c|}
\hline \multirow[t]{3}{*}{ Symptom* } & \multicolumn{4}{|c|}{ Posterior mean $(95 \% \mathrm{Crl})^{+}$} \\
\hline & \multicolumn{2}{|c|}{$\begin{array}{l}\text { FFR with EV and FFR with EV+AVS vs. } \\
\text { standard FFR }\end{array}$} & \multicolumn{2}{|c|}{$\begin{array}{l}\text { FFR with EV+AVS vs. } \\
\text { FFR with EV }\end{array}$} \\
\hline & After 2 hr & After $12 \mathrm{hr}$ & After $2 \mathrm{hr}$ & After $12 \mathrm{hr}$ \\
\hline \multicolumn{5}{|l|}{ Headache } \\
\hline FFR alone ${ }^{\ddagger}$ & 0.00 & 0.00 & - & - \\
\hline FFR with EV & $-0.35(-0.66$ to -0.05$)$ & $-0.30(-0.62$ to 0.00$)$ & 0.00 & 0.00 \\
\hline FFR with EV+AVS & $-0.87(-1.19$ to -0.56$)$ & $-0.76(-1.07$ to -0.46$)$ & $-0.52(-0.84$ to -0.20$)$ & $-0.46(-0.76$ to -0.14$)$ \\
\hline \multicolumn{5}{|l|}{ Giddiness } \\
\hline FFR alone ${ }^{*}$ & 0.00 & 0.00 & - & - \\
\hline FFR with EV & $-0.39(-0.70$ to -0.08$)$ & $-0.28(-0.59$ to 0.02$)$ & 0.00 & 0.00 \\
\hline FFR with EV+AVS & $-0.97(-1.29$ to -0.65$)$ & $-0.78(-1.09$ to -0.47$)$ & $-0.58(-0.90$ to -0.26$)$ & $-0.49(-0.76$ to -0.14$)$ \\
\hline \multicolumn{5}{|l|}{ Tiredness } \\
\hline FFR alone $e^{\ddagger}$ & 0.00 & 0.00 & - & - \\
\hline FFR with EV & $-0.62(-0.93$ to -0.32$)$ & $-0.64(-0.95$ to -0.34$)$ & 0.00 & 0.00 \\
\hline FFR with EV+AVS & $-0.99(-1.30$ to -0.68$)$ & $-0.96(-1.26$ to -0.65$)$ & $-0.37(-0.68$ to -0.05$)$ & $-0.32(-0.63$ to -0.01$)$ \\
\hline \multicolumn{5}{|l|}{ Difficulty breathing } \\
\hline FFR alone & 0.00 & 0.00 & - & - \\
\hline FFR with EV & $-0.62(-0.91$ to -0.33$)$ & $-0.52(-0.81$ to -0.23$)$ & 0.00 & 0.00 \\
\hline FFR with EV+AVS & $-1.26(-1.56$ to -0.97$)$ & $-1.22(-1.52$ to -0.92$)$ & $-0.65(-0.94$ to -0.36$)$ & $-0.69(-0.99$ to -0.40$)$ \\
\hline \multicolumn{5}{|l|}{ Difficulty walking } \\
\hline FFR alone ${ }^{*}$ & 0.00 & 0.00 & - & - \\
\hline FFR with EV & $-0.46(-0.79$ to -0.13$)$ & $-0.36(-0.68$ to -0.04$)$ & 0.00 & 0.00 \\
\hline FFR with EV+AVS & $-0.80(-1.14$ to -0.48$)$ & $-0.62(-0.95$ to -0.29$)$ & $-0.34(-0.68$ to 0.00$)$ & $-0.26(-0.60$ to 0.08$)$ \\
\hline \multicolumn{5}{|c|}{$\begin{array}{l}\text { Sensation of warmth within } \\
\text { the mask }\end{array}$} \\
\hline FFR alone $e^{*}$ & 0.00 & 0.00 & - & - \\
\hline FFR with EV & $-0.68(-0.97$ to -0.40$)$ & $-0.52(-0.81$ to -0.23$)$ & 0.00 & 0.00 \\
\hline FFR with EV+AVS & $-1.48(-1.79$ to -1.18$)$ & $-1.41(-1.72$ to -1.11$)$ & $-0.80(-1.08$ to -0.51$)$ & $-0.89(-1.18$ to -0.59$)$ \\
\hline \multicolumn{5}{|c|}{ Sweating within the mask } \\
\hline FFR alone ${ }^{*}$ & 0.00 & 0.00 & - & - \\
\hline FFR with EV & $-0.64(-0.92$ to -0.35$)$ & $-0.63(-0.92$ to -0.34$)$ & 0.00 & 0.00 \\
\hline FFR with EV+AVS & $-1.30(-1.60$ to -1.00$)$ & $-1.29(-1.60$ to -0.99$)$ & $-0.67(-0.95$ to -0.38$)$ & $-0.66(-0.95$ to -0.37$)$ \\
\hline \multicolumn{5}{|c|}{ Itchiness within the mask } \\
\hline FFR alone $e^{*}$ & 0.00 & 0.00 & - & - \\
\hline FFR with EV & $-0.69(-0.98$ to -0.39$)$ & $-0.69(-0.98$ to -0.39$)$ & 0.00 & 0.00 \\
\hline FFR with EV+AVS & $-0.95(-1.25$ to -0.66$)$ & $-1.10(-1.41$ to -0.80$)$ & $-0.27(-0.56$ to 0.03$)$ & $-0.41(-0.72$ to -0.12$)$ \\
\hline \multicolumn{5}{|c|}{$\begin{array}{l}\text { Uncomfortable tightness of } \\
\text { mask seal }\end{array}$} \\
\hline FFR alone ${ }^{*}$ & 0.00 & 0.00 & - & - \\
\hline FFR with EV & $-0.76(-1.05$ to -0.48$)$ & $-0.88(-1.17$ to -0.58$)$ & 0.00 & 0.00 \\
\hline FFR with EV+AVS & $-1.33(-1.63$ to -1.04$)$ & $-1.34(-1.64$ to -1.04$)$ & $-0.57(-0.86$ to -0.27$)$ & $-0.46(-0.75$ to -0.17$)$ \\
\hline \multicolumn{5}{|l|}{ Discomfort } \\
\hline FFR alone ${ }^{\ddagger}$ & 0.00 & 0.00 & - & - \\
\hline FFR with EV & $-0.72(-1.00$ to -0.44$)$ & $-0.77(-1.06$ to -0.48$)$ & 0.00 & 0.00 \\
\hline FFR with EV+AVS & $-1.13(-1.41$ to -0.85$)$ & $-1.26(-1.55$ to -0.96$)$ & $-0.40(-0.69$ to -0.12$)$ & $-0.48(-0.77$ to -0.20$)$ \\
\hline \multicolumn{5}{|l|}{ Exertion } \\
\hline FFR alone ${ }^{*}$ & 0.00 & 0.00 & - & - \\
\hline FFR with EV & $-0.47(-0.76$ to -0.19$)$ & $-0.45(-0.74$ to -0.16$)$ & 0.00 & 0.00 \\
\hline FFR with EV+AVS & $-0.80(-1.09$ to -0.51$)$ & $-1.18(-1.47$ to -0.89$)$ & $-0.34(-0.62$ to -0.05$)$ & $-0.73(-1.01$ to -0.44$)$ \\
\hline
\end{tabular}

*Symptoms, comfort and exertion were rated on a 5-point Likert scale (1: less symptoms/discomfort/exertion; 5: most symptoms/discomfort/exertion). +Posterior means with $95 \% \mathrm{Crl}$ of the regression coefficients of symptoms, discomfort or exertion when compared to the reference group and $95 \% \mathrm{Crl}$ were determined using a multivariate ordered probit mixed-effects model of the distribution of the Likert scores of symptoms, perceived comfort and perceived exertion over the three sampling periods (at baseline, after $2 \mathrm{hr}$ of standardised activity and after $12 \mathrm{hr}$ of mask usage) $¥$ Standard FFR (without EV or AVS) was used as the reference group. AVS: active venting system; Crl: credible interval; EV: exhalation valve; FFR: filtering facepiece respirator 


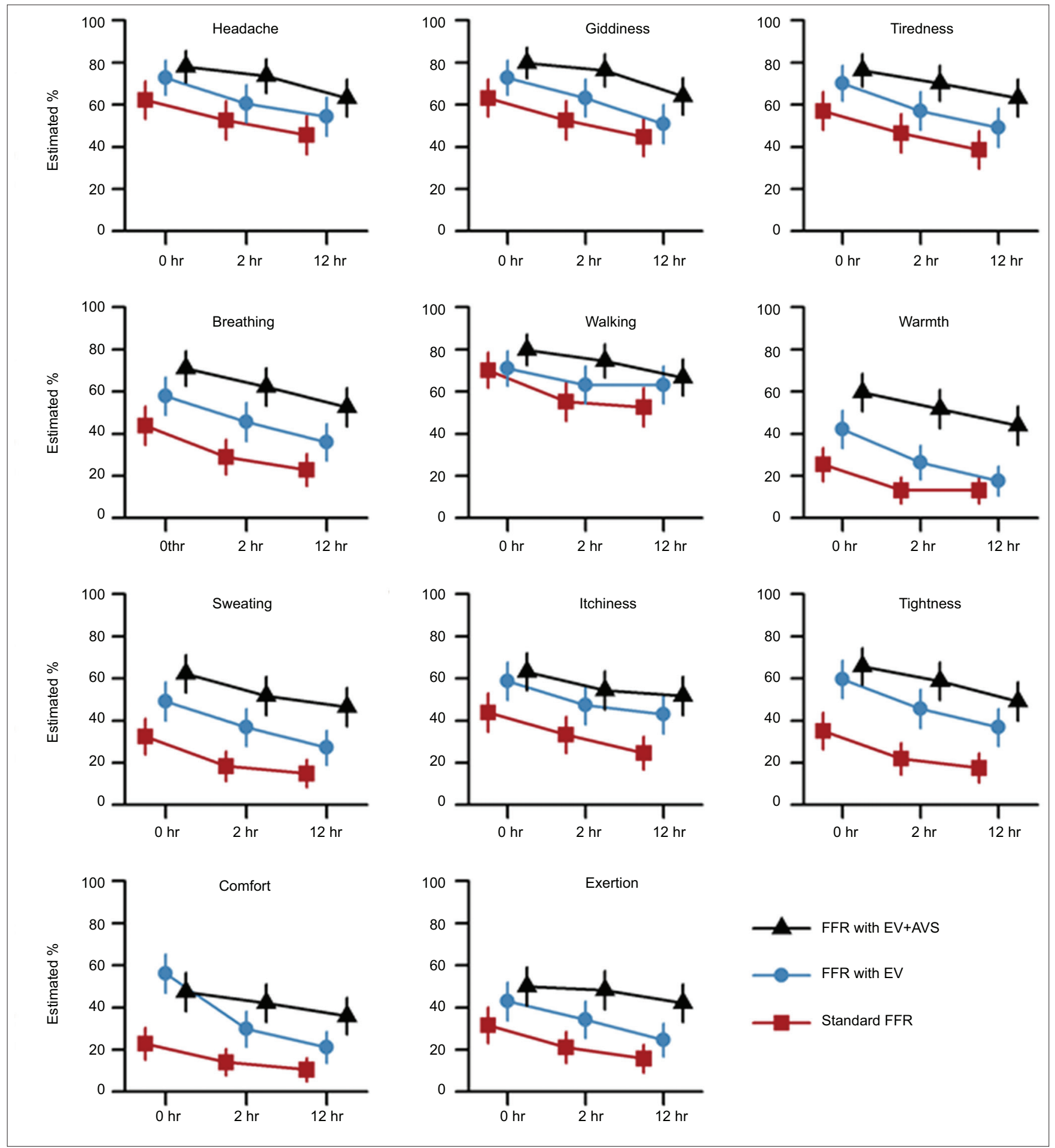

Fig. 3 Charts show summary of estimated percentages of score $\leq 2$ with $95 \%$ credible interval of participants having less perceived symptoms, discomfort and exertion associated with the usage of various filtering facepiece respirator (FFR) options, with and without exhalation valve (EV) or active venting system (AVS), at two hours and 12 hours $(n=114)$. Pr: estimated percentage

compared to standard FFR, as they experienced less symptoms, discomfort and exertion with the former two options. The actual Likert scores for the majority of symptoms were not normally distributed. However, the statistical model developed proved a good fit for the observed data, with substantial overlaps between 95\% Crl and observed distributions in all Likert score intervals. Generally, there was an increase in the average Likert scores with increase in the length of time of wearing the mask for each FFR option.
Overall, at the two-hour and 12-hour time points, both FFR with EV and FFR with EV+AVS were significantly more comfortable than standard FFR. FFR with EV+AVS was also significantly more comfortable than FFR with EV alone. At the two-hour and 12-hour time points, both FFR with EV and FFR with $\mathrm{EV}+\mathrm{AVS}$ led to significantly less perceived exertion than standard FFR, and FFR with EV+AVS led to significantly less perceived exertion than FFR with EV alone. Perceived symptom severity, exertion and discomfort were significantly associated with long 
duration of wearing standard FFR (posterior mean at two hours: 0.33-0.69; posterior mean at 12 hours: $0.62-0.91$ ).

\section{DISCUSSION}

This study aimed to assess user discomfort and exertion after wearing different FFRs during the performance of outdoor essential duties. FFR with EV performed significantly better than standard FFR in terms of reducing user-perceived symptoms, discomfort and exertion. This supported the evidence presented in the existing literature, ${ }^{(12)}$ where the physical benefits of incorporating an EV into FFR (e.g. decreased exhalation resistance, improved dead space heat dissipation) were translated into actual improvements in user-perceived comfort.

Notably, to the best of our knowledge, this is the first trial of FFRs incorporating AVS, which is a new additional feature in FFRs that were already using EVs. As the various FFRs used in our study were purchased by the SAF, there was no conflict of interest. Our findings demonstrated that the attachment of the microventilator resulted in improved symptoms. For prolonged use, FFR with EV+AVS also had significantly less exertion and discomfort compared to FFR with EV alone. This is especially relevant to occupations where outdoor work cannot be avoided, such as in the military. We believe that the improvements noted in the symptoms were likely due to improved ventilation of the dead space within the FFR, which was provided by the AVS. Apart from ventilating the dead space, as per fluid dynamics modelling, the venting of air through the blower in FFR with EV+AVS resulted in the sucking out of air away from the FFR, thus reducing the likelihood of rebreathing $\mathrm{CO}_{2}$ when compared to the standard FFR. ${ }^{(13)}$ These improvements did not come at the expense of other symptoms, such as increased difficulty in walking or tightness of mask seal, which might have been expected due to the slight increase in the FFR's weight on account of the AVS.

Personnel can be exposed to particulate-based air pollution in a number of ways. In recent military deployments in Iraq and Afghanistan, potential sources of particulates included the open-air burning of refuse, which can negatively affect the cardiorespiratory health of personnel. ${ }^{(19,20)}$ In addition, deployment in areas with high levels of air pollution at baseline can also impair the cardiorespiratory performance of personnel. ${ }^{21}$ While respiratory protection can be achieved via engineering and administrative controls, this may not always be possible. Thus, the usage of appropriate FFRs as PPE is important to protect personnel from the effects of particulates, either from occupational exposure or due to deployment to areas with significant air pollution.

The improved comfort following modifications to the standard FFR could potentially encourage better compliance with PPE usage and enable better task performance without distractions due to increased discomfort or exertion. In a study of healthcare workers required to wear FFRs for two 12-hour shifts, almost a quarter of the respirator removals were reportedly due to discomfort experienced while wearing them. ${ }^{(22)}$ Another study found that workers were interested in FFRs that interfered less with breathing and reduced the build-up of heat. ${ }^{(23)}$ Our findings that the incorporation of an AVS into the FFR resulted in the reduction of symptoms, exertion and discomfort may prompt improved compliance with PPE among workers who are required to use FFRs for prolonged periods. At present, however, it is not known if FFR with $\mathrm{EV}$, with or without an AVS, can be used for infection control in the healthcare setting, especially since AVS is not meant to be disposable and disinfection after healthcare procedures would pose a challenge. Future studies should look into the use of these technologies for infection control and protection.

Our study had some limitations, which included the fact that participants were not blinded to the type of FFR they were using at each point in time. It was also not possible to blind them on the presence or absence of an EV and AVS on FFRs, given that these were integral components of the mask. Next, only user-perceived discomfort, exertion and symptoms were measured in conjunction with the use of FFRs, and physiological measurements, such as heart rate and blood pressure, were not taken. Also, our trial was conducted when air quality was not severe enough to warrant the use of FFRs. That notwithstanding, it can be safely assumed that results would be similar for the use of FFR during air pollution events. Finally, the study was conducted among young men. In Singapore, national service is compulsory, and for this reason, our participants can be taken to reflect the general local population in this particular demographic segment. Among older people, on the other hand, we could expect perceived symptoms to be greater and hence improvement in symptoms with the use of FFR with EV+AVS could be potentially greater as well.

In conclusion, usage of FFR with EV+AVS resulted in significant reductions in perceived discomfort, exertion and most symptoms, when compared to both FFR with EV and standard FFR. FFR with EV fared significantly better in terms of perceived discomfort, exertion and most symptoms than standard FFR. Further trials are necessary to determine whether these perceived improvements correspond to objectively measurable physiological benefits and improvements in compliance with FFR usage, especially among military personnel and healthcare workers, who may need to use FFRs for prolonged periods of time and for essential duties.

\section{ACKNOWLEDGEMENTS}

This study was funded by the SAF as an operational evaluation. The authors did not receive any funding, salary, grants or honoraria from the manufacturers or distributors of the respirators used in the study. Xiahong Zhao and Alex R Cook are supported by the Centre for Infectious Disease Epidemiology and Research, Saw Swee Hock School of Public Health, National University of Singapore, National University Health System, Singapore. We thank the Medical Corps of SAF and 2nd People's Defence Force for providing the resources to organise and support our study.

\section{REFERENCES}

1. Pope CA 3rd, Burnett RT, Thun MJ, et al. Lung cancer, cardiopulmonary mortality, and long-term exposure to fine particulate air pollution. JAMA 2002; 287:1132-41.

2. Xu J, Tai X, Betha R, He J, Balasubramanian R. Comparison of physical and chemical properties of ambient aerosols during the 2009 haze and non-haze periods in Southeast Asia. Environ Geochem Health 2015; 37:831-41.

3. Betha R, Behera SN, Balasubramanian R. 2013 Southeast Asian smoke haze: 
fractionation of particulate-bound elements and associated health risk. Environ Sci Technol 2013; 48:4327-35.

4. Emmanuel SC. Impact to lung health of haze from forest fires: the Singapore experience. Respirology 2000; 5:175-82.

5. Awang MB, Jaafar AB, Abdullah AM, et al. Air quality in Malaysia: impacts, management issues and future challenges. Respirology 2000; 5:183-96.

6. Ministry of Health, Singapore. FAQ: Impact of Haze on Health. Available at: https:/ www.moh.gov.sg/content/moh_web/home/pressRoom/Current_Issues/2014/haze/ faq--impact-of-haze-on-healtho.html. Accessed September 15, 2016.

7. Martyny J, Glazer CS, Newman LS. Respiratory protection. N Engl J Med 2002; 347:824-30.

8. Langrish JP, Mills NL, Chan JK, et al. Beneficial cardiovascular effects of reducing exposure to particulate air pollution with a simple facemask. Part Fibre Toxicol 2009; 6:8.

9. Roberge RJ, Coca A, Williams WJ, Powell JB, Palmiero AJ. Physiological impact of the N95 filtering facepiece respirator on healthcare workers. Respir Care 2010; 55:569-77.

10. Roberge RJ, Kim JH, Benson S. N95 filtering facepiece respirator deadspace temperature and humidity. J Occup Environ Hyg 2012; 9:166-71.

11. Shenal BV, Radonovich LJ Jr, Cheng J, Hodgson M, Bender BS. Discomfort and exertion associated with prolonged wear of respiratory protection in a health care setting. J Occup Environ Hyg 2012; 9:59-64.

12. Roberge RJ. Are exhalation valves on N95 filtering facepiece respirators beneficial at low-moderate work rates: an overview. J Occup Environ Hyg 2012; 9:617-23.

13. Birgersson E, Tang EH, Lee WL, Sak KJ. Reduction of carbon dioxide in filtering facepiece respirators with an active-venting system: a computational study. PLoS One 2015; 10:e0130306.
14. Daykin AR, Moffatt PG. Analyzing ordered responses: a review of the ordered probit model. Understanding Stat 2010; 1:157-66.

15. Putt $M$, Chinchilli VM. A mixed effects model for the analysis of repeated measures cross-over studies. Stat Med 1999; 18:3037-58.

16. Geweke J. Evaluating the accuracy of sampling-based approaches to the calculation of posterior moments. In: Bernardo JM, Berger JO, Dawid AP, Smith AF, eds. Bayesian Statistics 4: Proceedings of the Fourth Valencia International Meeting. Oxford: Oxford University Press, 1992: 163-93.

17. Plummer M, Stukalov A, Denwood, M. Bayesian graphical models using MCMC. Available at: https://cran.r-project.org/web/packages/rjags/index.html. Accessed September 15, 2016.

18. R: A language and environment for statistical computing. Available at: http:// www.R-project.org/. Accessed September 15, 2016.

19. Szema AM. Occupational lung diseases among soldiers deployed to Iraq and Afghanistan. Occup Med Health Aff 2013; 1.

20. Weese $\mathrm{CB}$, Abraham JH. Potential health implications associated with particulate matter exposure in deployed settings in Southwest Asia. Inhal Toxicol 2009; 21:291-6.

21. Oliveira RS, Barros Neto TL, Braga AL, et al. Impact of acute exposure to air pollution on the cardiorespiratory performance of military firemen. Braz J Med Biol Res 2006; 39:1643-9.

22. Rebmann T, Carrico R, Wang J. Physiologic and other effects and compliance with long-term respirator use among medical intensive care unit nurses. Am J Infect Control 2013; 41:1218-23.

23. Baig AS, Knapp C, Eagan AE, Radonovich LJ Jr. Healthcare workers' views about respirator use and features that should be included in the next generation of respirators. Am J Infect Control 2010; 38:18-25. 